\title{
Minimal Residual Disease in Neovascular Age-Related Macular Degeneration
}

\section{Minimal Residual Disease bei altersbedingter Makuladegeneration}

\author{
Authors \\ Jakob Siedlecki $^{10}$, Jose Pulido ${ }^{2,3}$, Siegfried Priglinger $^{1}$ \\ Affiliations \\ 1 Augenklinik der LMU, Klinikum der Universität München, \\ München, Germany \\ 2 Department of Translational Ophthalmology, Wills Eye \\ Hospital, Philadelphia, Pennsylvania, United States \\ 3 Ophthalmology and Molecular Medicine, Mayo Clinic, \\ Rochester, MN (Minnesota), United States
}

Key words

minimal residual disease, complete remission, age-related macular degeneration, AMD, choroidal neovascularization, CNV

\author{
Schlüsselwörter \\ Minimal residual Disease, OCT, altersbedingte Makula- \\ degeneration (Age-related macular Degeneration), OCT

$\begin{array}{ll}\text { received } & 4.11 .2020 \\ \text { accepted } & 27.11 .2020 \\ \text { published online } & 14.4 .2021\end{array}$ \\ Bibliography \\ Klin Monatsbl Augenheilkd 2021; 238: 700-702 \\ DOI 10.1055/a-1333-3522 \\ ISSN 0023-2165 \\ (c) 2021. Thieme. All rights reserved. \\ Georg Thieme Verlag KG, Rüdigerstraße 14, \\ 70469 Stuttgart, Germany
}

Correspondence

Dr. Jakob Siedlecki

Klinikum der Universität München, Augenklinik der LMU

Mathildenstraße 8, 80336 München, Germany

Phone: + 498951603811 , Fax: + 498951604559

jakob.siedlecki@med.uni-muenchen.de

\section{ABSTRACT}

With the advent of long-acting anti-vascular endothelial growth factor substances, "healing of AMD (age-related macular degeneration)" might be easily assumed due to prolonged treatment intervals. Adoption of the oncological concept of minimal residual disease for quiescent choroidal neovascularization (CNV) lesions might enhance the importance of continued monitoring, and spur research into the core of the disease, i.e., CNV biology.

\section{ZUSAMMENFASSUNG}

Mit der Zulassung langwirksamer Anti-VEGF-Substanzen könnte in Zukunft der Eindruck beim Patienten entstehen, eine neovaskuläre altersbedingte Makuladegeneration sei "geheilt", da lange Intervalle zwischen den einzelnen Behandlungen Realität geworden sind. Die Einführung des onkologischen Konzeptes „Minimal residual Disease“ (MRD) könnte hier die Bedeutung quiescenter, unter Behandlung nicht exsudativer choroidaler Neovaskularisationen wieder mehr in den Fokus rücken, um auf die Wichtigkeit des langfristigen Krankheitsmonitorings hinzuweisen.

\section{Dear Editor,}

In any oncologic treatment, the ultimate goal of therapy is to heal the patient by eliminating every single tumor cell of the malignancy involved. Due to the inability to screen for treatment efficacy at a cellular level, complete eradication of tumor cells, however, cannot be proven. As a substitute, complete remission (CR) is being widely used as another ultimate goal of therapy, defined as a total absence of tumor mass on sensitive radiologic imaging (e.g., CT, MRI, or PET/CT) or histology (e.g., cellular morphology on blood smear in leukemia) [1]. As a major shortcoming, the concept of CR, however, cannot completely exclude future recur- rence, as even one single remaining malignant cell can theoretically induce tumor regrowth.

To explain the discrepancy between clinical/radiographic CR and the immediate reality of relapse, the term minimal residual disease (MRD) has been introduced. MRD is defined as the residuum of cancer cells remaining in the human body in spite of successful treatment inducing $C R$, freeing the patient from symptoms and making the tumor "disappear" on conventional clinical testing, although malignancy still persists in a very low, often undetectable quantity [1]. In medical oncology, MRD is then subdivided into undetectable MRD (UMRD) and MRD where UMRD is 
the disease that is present at a level lower than can be found by present methods of detection.

Beyond its philosophical aspect explaining recurrence in spite of CR, the concept of MRD is a result of the continuous struggle to improve sensitivity in screening for residual tumor traces. As a term, MRD was first described in leukemia, defined as a state of disease only detectable by laboratory techniques more sensitive than the previous state-of-the-art assessment of cellular morphology, i.e., using novel tools like flow cytometry or polymerase chain reaction [2]. Therefore, the final implicit answers to pivotal questions of disease control might be strongly dependent on the sensitivity of the test screening for MRD: Is the tumor completely eradicated? What is the prognosis of the patient, especially concerning recurrence? Which changes during disease monitoring should warrant additional treatment?

Recently, Stacey and Pulido have advocated the use of the term minimal residual disease in ocular oncology [3]. In ocular oncology, MRD is now proposed to be used for the rare cells in the vitreous that can be present following intensive treatment of the eye in vitreoretinal lymphoma [3]. In uveal melanoma it has been proposed as the term for patients where an eye has been treated and imaging does not show detectable disease [3].

But even beyond oncology, recent technological advances in ocular imaging have strikingly disrupted the pathophysiological understanding of retinal disease so profoundly that parallels can easily be drawn between the advent of flow cytometry in leukemia diagnostics, necessitating the new term of MRD, and the multimodal detection of subclinical, asymptomatic disease features previously going unnoticed on a regular dilated fundus exam, now warranting the clinician's attention to assess their (future) significance. For example, Gantner et al. have recently shown that relatives of patients with telangiectasia macular II (MacTel II) exhibit specific pathologic phenotypes of MacTel II as seen on fluorescence lifetime imaging ophthalmoscopy, although the patients neither show symptoms nor exhibit specific findings suggestive of the disease on standard retinal imaging [4]. Additionally, in regard to the concept of MRD, Querques et al., using fluorescein angiography (FA), and Carnevali et al., using optical coherence tomography (OCT) angiography, have recently described the detection of choroidal neovascularization (CNV) lesions that neither show active exudation on FA nor any fluid on OCT, be it sub-RPE, sub- or intraretinal [5,6]. Nevertheless, these asymptomatic, non-exudative seemingly quiescent CNVs show biological activity by subsequent lesion growth [7] and can, moreover, convert into actively exudative lesions requiring anti-vascular endothelial growth factor (VEGF) treatment [8]. With the increasing availability of OCT angiography worldwide, it has become evident that these subclinical lesions are much more frequent than previously estimated.

In any anti-VEGF treatment for neovascular age-related macular degeneration (nAMD), the ultimate goal is to heal the patient by "drying" the macula, i.e., achieve inactivation of the underlying CNV, and thus prevent macular fluid, hemorrhage, and fibrosis. It is well known that due to the astounding chronicity of CNV in nAMD, anti-VEGF treatment has to be applied over years with variable time frames between last injection and relapse on OCT [9-11]. Of course, some patients will develop significant visually disabling fibrosis and atrophy, which seem to inactivate previous
CNV lesions, and treatment will eventually be stopped in these patients. On the other hand, some patients will maintain a "dry macula" without further treatment after a variable amount of previous injections, suggesting that "nAMD has been healed". For these patients, various "stop" protocols have been suggested, implemented in established "treat and extend" regimens [9-11]. While some authors' stop therapy after patients maintain a dry macula after the first 12-week interval [9], others suggest demanding three consecutive 12-week intervals without reactivation on OCT [10], and others even suggest three consecutive 16-week intervals [11].

So, are these patients healed? Unfortunately, as tumors show recurrence, $\mathrm{nAMD}$ relapses recur even after the most demanding treat and extend stop protocol. Nguyen et al., stopping treatment after the first successful 12-week interval, reported recurrence of lesion activity on OCT in $41 \%$ of eyes in the first year, a percentage that rises to $79 \%$ at year 5 , with a median time to reactivation of 1.38 years [9]. Adrean et al., stopping treatment after three successful 12-week intervals, reported a $29.4 \%$ recurrence rate after a mean of 1.2 years [10]. Arendt et al., stopping after three successful 16-week intervals, reported a $13 \%$ recurrence rate after a mean of 0.7 years [11].

According to these data, patients have to be informed that after stopping current anti-VEGF substances for nAMD, their likelihood of disease reactivation can be up to $80 \%$ in 5 years - which is a number that is worse than some cancer recurrence rates at 5 years [12]. Miere et al. have recently shown that up to $94 \%$ of fibrotic macular lesions after CNV treatment show residual flow on OCT angiography [13], while Capuano et al. suggest that the prevalence of CNV even in late-stage geographic atrophy seems to be much higher than previously thought [8].

The same way we adopted anti-VEGF treatment from oncologists 13 years ago, it might be time to also translate their terminology into our specialty of ophthalmology. The term minimal residual disease fits with the concept that CNV lesions persist in spite of years-long treatment, and, in spite of the absence of CNV activity on OCT. These residual CNV membranes have a risk of reactivation, damaging visual acuity to an extent that might not be improved by resumption of therapy. In the study by Nguyen et al., a mean loss of 4.2 letters is reported secondary to disease reactivation after the first treatment regimen [9]. Although these eyes regained 1.2 letters at 12 months, the net loss due to reactivation was 3.3 letters [9].

Are these patients with recurrence treated too late? If we translate the concept of CR into our setting, CR represents the absence of disease activity, mostly fluid, on OCT, and this is the current stimulus to reinitiate treatment. If we wait for $C R$ to resume treatment, are we allowing the induction of damage before we reinject?

As many authors suggest, monitoring patients following the cessation of treatment to resume treatment is essential [9-11]. The concept then that the CNV is still present as MRD might be an important new concept for the retina specialist as well as for educating the patient. The same way that quiescent tumor cells can resume proliferating, quiescent endothelial cells in CNV might react to a changing milieu by resuming migration, proliferation, 
and tube formation, thus reactivating leakage and destructive growth.

In oncology, directly targeting MRD has been shown to improve outcomes compared to waiting for a failure of CR, i.e, tumor recurrence on radiologic imaging [1]. If we as ophthalmologists seek to improve our outcomes, it might be reasonable to develop methods to screen for MRD prior to exudation, allowing for treatment before destructive growth and leakage can commence.

Actively sprouting neovessels can now be distinguished from matured, established lesions [14]. To date, OCT angiography is, however, still failing to provide easily recognizable biomarkers predicting CNV activity, which ultimately results in fluid on OCT. To improve long-term outcomes, technical improvements as well as explorative prospective studies are warranted to take CNV out of the black box of MRD and improve the understanding of its biology.

Quiescent, non-exudative CNV might promote protection against the progression of macular atrophy [15]. With this new approach, accepting and modulating CNV as MRD to hinder destructive growth, but foster trophic effects, will probably become an important future direction in the treatment of $\mathrm{nAMD}$, in which OCT angiography or newer modalities will hopefully play a decisive role.

Longer-acting anti-VEGF substances prolonging the interval between injections are being approved and patients and caregivers might be tempted to more easily assume the disease has been healed, merely because the interval between injections can be extended beyond a few months. With the concept of CNV as MRD in mind, it will be the ophthalmologist's duty to educate patients about the chronicity of their disease, their MRD they are involuntarily carrying, and the importance of compliance to maintain the visual acuity gains introduced by the short-acting antiVEGF substance revolution also in this new, long-acting anti-VEGF evolution. On the other hand, it will be our duty to improve existing technology and invent new methods to monitor MRD, preventing regrowth and exudation before photoreceptors are damaged, and thereby favorably guide MRD in AMD. As we push the limits for now and in the future, it is worth continuing to squeeze down nAMD MRD further to the very limit where we will be actually able to tell a patient that they have truly been cured.

\section{Conflict of Interest}

No author has any financial disclosures relevant to this manuscript J.S. has received speaker fees and/or travel expenses from Novartis Pharma GmbH, Carl Zeiss Meditec AG, Bayer AG, Oculentis OSD Medical $\mathrm{GmbH}$, and Pharm-Allergan $\mathrm{GmbH}$. J. S. P. has been awarded patents on viral oncology and has submitted patents on induced pluripotent stem cell-derived retinal pigment epithelial cells (iPSC-RPE) and is a partial owner of LAGEN Laboratories that makes iPSC-RPE for in vitro use. S. G.P. has received speaker fees and/or travel expenses from Novartis Pharma GmbH, Oertli AG, Bayer AG, Alcon Pharma GmbH, and PharmAllergan $\mathrm{GmbH}$.

\section{References}

[1] Luskin MR, Murakami MA, Manalis SR et al. Targeting minimal residual disease: a path to cure? Nat Rev Cancer 2018; 18: 255-263. doi:10.1038/nrc.2017.125

[2] Paietta E. Assessing minimal residual disease (MRD) in leukemia: a changing definition and concept? Bone Marrow Transplant 2002; 29 : 459-465. doi:10.1038/sj.bmt.1703388

[3] Stacey AW, Pulido JS. The Concept of Minimal Residual Disease in the Treatment and Staging of Vitreoretinal Lymphoma. Retina 2020; 40: 1213-1214. doi:10.1097/iae.0000000000002851

[4] Gantner ML, Eade K, Wallace M et al. Serine and Lipid Metabolism in Macular Disease and Peripheral Neuropathy. N Engl J Med 2019; 381: 1422-1433. doi:10.1056/NEJMoa1815111

[5] Carnevali A, Cicinelli MV, Capuano V et al. Optical Coherence Tomography Angiography: A Useful Tool for Diagnosis of Treatment-Naive Quiescent Choroidal Neovascularization. Am J Ophthalmol 2016; 169: 189198. doi:10.1016/j.ajo.2016.06.042

[6] Querques G, Srour M, Massamba N et al. Functional characterization and multimodal imaging of treatment-naïve "quiescent" choroidal neovascularization. Invest Ophthalmol Vis Sci 2013; 54: 6886-6892. doi:10.1167/iovs.13-11665

[7] Carnevali A, Sacconi R, Querques L et al. Natural History of TreatmentNaïve Quiescent Choroidal Neovascularization in Age-Related Macular Degeneration Using OCT Angiography. Ophthalmol Retina 2018; 2: 922-930. doi:10.1016/j.oret.2018.02.002

[8] Capuano V, Miere A, Querques L et al. Treatment-Naive Quiescent Choroidal Neovascularization in Geographic Atrophy Secondary to Nonexudative Age-Related Macular Degeneration. Am J Ophthalmol 2017; 182: 45-55. doi:10.1016/j.ajo.2017.07.009

[9] Nguyen V, Vaze A, Fraser-Bell $S$ et al. Outcomes of Suspending VEGF Inhibitors for Neovascular Age-Related Macular Degeneration When Lesions Have Been Inactive for 3 Months. Ophthalmol Retina 2019; 3: 623-628. doi:10.1016/j.oret.2019.05.013

[10] Adrean SD, Chaili S, Grant S et al. Recurrence Rate of Choroidal Neovascularization in Neovascular Age-Related Macular Degeneration Managed with a Treat-Extend-Stop Protocol. Ophthalmol Retina 2018; 2: 225230. doi:10.1016/j.oret.2017.07.009

[11] Arendt P, Yu S, Munk MR et al. Exit strategy in a treat-and-extend regimen for exudative age-related macular degeneration. Retina 2019; 39: 27-33. doi:10.1097/iae.0000000000001923

[12] Mahvi DA, Liu R, Grinstaff MW et al. Local Cancer Recurrence: The Realities, Challenges, and Opportunities for New Therapies. CA Cancer J Clin 2018; 68: 488-505. doi:10.3322/caac.21498

[13] Miere A, Semoun O, Cohen SY et al. Optical coherence tomography angiography features of subretinal fibrosis in age-related macular degeneration. Retina 2015; 35: 2275-2284. doi:10.1097/iae. 0000000000000819

[14] Perrott-Reynolds R, Cann R, Cronbach N et al. The diagnostic accuracy of OCT angiography in naive and treated neovascular age-related macular degeneration: a review. Eye (Lond) 2019; 33: 274-282. doi:10.1038/ s41433-018-0229-6

[15] Pfau M, Möller PT, Künzel SH et al. Type 1 Choroidal Neovascularization Is Associated with Reduced Localized Progression of Atrophy in AgeRelated Macular Degeneration. Ophthalmol Retina 2020; 4: 238-248. doi:10.1016/j.oret.2019.09.016 